\title{
GestureWrist and GesturePad: Unobtrusive Wearable Interaction Devices
}

\author{
Jun Rekimoto \\ Interaction Laboratory \\ Sony Computer Science Laboratories, Inc. \\ 3-14-13 Higashigotanda \\ Shinagawa-ku, Tokyo 141-0022, Japan \\ Phone: +81354484380 \\ Fax +81354484273 \\ rekimoto@acm.org \\ http://www.csl.sony.co.jp/person/rekimoto.html
}

\begin{abstract}
In this paper, we introduce two input devices for wearable computers, called GestureWrist and GesturePad. Both devices allow users to interact with wearable or nearby computers by using gesture-based commands. Both are designed to be as unobtrusive as possible, so they can be used under various social contexts. The first device, called GestureWrist, is a wristband-type input device that recognizes hand gestures and forearm movements. Unlike DataGloves or other hand gesture-input devices, all sensing elements are embedded in a normal wristband. The second device, called GesturePad, is a sensing module that can be attached on the inside of clothes, and users can interact with this module from the outside. It transforms conventional clothes into an interactive device without changing their appearance.
\end{abstract}

Keywords: Wearable computers, input technologies, gesture sensing, interactive clothing, on-body networking.

\section{Introduction}

Generally, wearable computers are expected to be continuously worn and support their users. To enable this goal, several technology and design challenges arise, including output technologies, such as head-up displays, and input technologies. Two main input types are being considered, one is implicit input by contextual sensing such as location information, and the other is explicit input from a user. In this paper, we focus on the latter type.

For wearable computers to be used in actual everyday situations, we consider the following features to be important:
Support hands-free operations or allow quick changes between normal and operation modes:

Some input devices used with wearable computers need to be hold during operation. For example, "Twiddler" [2], a popular single-hand keyboard, requires the user to hold it to operate it during operation, and substantial time is required to correctly grip it. With one hand already occupied, performing two-handed tasks, which often occurs in actual situations, becomes difficult. Similarly, DataGlove [13], CyberGlove [11], or other glove-type input devices (such as [6]) also hinder performing real world tasks.

\section{Social acceptance:}

Input devices should be as natural and (conceptually) unnoticeable as possible for use in various social settings. Many current wearable input devices look unusual, or too "hi-tech", and often prevent normal social activities, such as shaking hands. Even though it is functional for the purpose of demonstration, it often becomes troublesome, even for the user, to wear it long-term in everyday situations. In contrast, traditional wearable accessories, such as wristwatches, eyeglasses, jewelry, and clothes are a vital part of our life style and are designed for comfortable long-time use. Wearable devices should be as acceptable as today's accessories, or they should be a part of these things.

In other words, we believe "unobtrusiveness" of input devices is essential for them to be used in everyday situations. One possible way to design such devices is to embed input sensors to conventional wearable items, such as wristwatches or clothing. Such input devices could be used to control worn computers or digital devices (such as cellular phones, PDAs, or MP3 players), or nearby computers and devices (such as presentation systems).

In this paper, we introduce two example devices, called GestureWrist and GesturePad. 
The first device, GestureWrist, is a wristwatch-type input device that recognizes human hand gestures by capacitively measuring changes in wrist shape. Combined with an acceleration sensor, which is also mounted to the wristband, the GestureWrist can be used as a command-input device, with a physical appearance almost identical to today's wristwatches.

The latter device, GesturePad, is a layer of sensor electrodes that transforms conventional clothes into interaction devices, or "interactive clothing". This module can be attached to an area of clothes such as a sleeve or a lapel. Also on capacitive sensing, it can detect and read finger motions applied to the outside of the clothing fabric, while shielding the capacitive influence from the human body.

\section{Related work}

Some wearable computers use physical dials, buttons, or touch-pads as input devices [10]. These devices are used to select menus or control nearby ubiquitous computers or appliances. We are aiming at similar applications by using more unobtrusive devices.

Baudel and Beaudouin-Lafon demonstrated a hand-gesture input system that is used as a remote control method [1]. A wearer can control a presentation system by using handgestures. Since this system is based on "DataGlove" and an attached position sensor, a user has to first put on a glove to use it. In contrast, our solution aims to be more seamless; using wearable input devices requires no particular preparation.

GesturePendant is a camera-based gesture recognition system that can be worn like a pendant [9]. A user can hand gesture in front of it while it is worn around the neck. The current prototype is still noticeably bigger than an ideal one, and would presupposedly always wear it over their clothes.

Wireless FingerRing is a hand-worn input device consisting of acceleration-sensitive finger rings and a wristbandtype receiver [3]. A user puts on four rings, and taps on a flat surface with one finger. This is detected by the ring's sensor, and the information is transmitted to the wristband receiver through an on-body network. Acceleration Sensing Glove also uses an acceleration sensor on each fingertip [6]. While wearing one finger ring is common and socially accepted, putting on four rings is unusual and thus it is unlikely all of us would do it. Supplying sufficient power to operate all the finger rings is an additional unsolved technical problem.

Measuring muscle tension (electromyogram, or EMG) and using the information as computer inputs has been widely studied [12]. This method is important for people with physical disabilities. However, it also involves some difficulties. One problem is placing the electrode. To correctly measure electricity, electrodes must have direct contact to the skin, often requiring wet-conductive gel. At least two (and often at least three) electrodes need to be attached to the skin, and maintain certain distances. These requirements make it difficult to configure a simple wristbandtype EMG sensor that can be easily worn. Our method measures the cross-sectional shape of the wrist, instead of using an EMG, to detect hand motions.

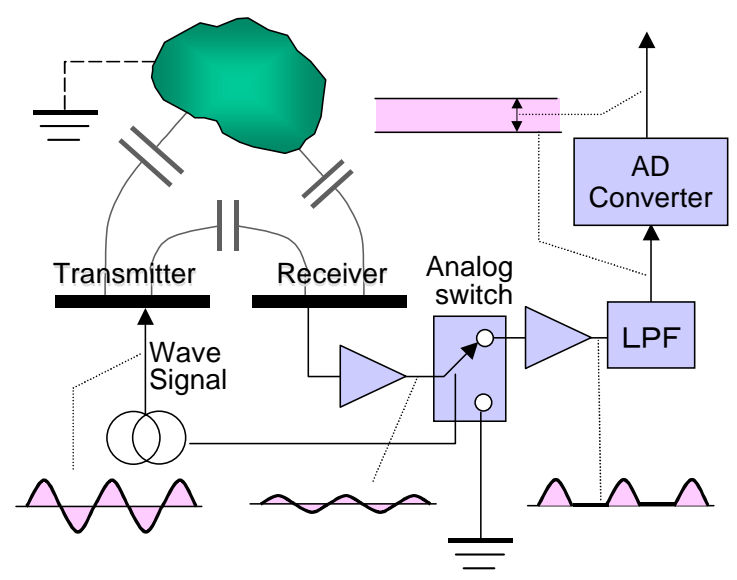

Figure 1: A capacitive sensor is used to measure distance between sensor electrodes and an object.

\section{Technological background}

Before describing our proposed input devices, we briefly introduce their sensing technologies.

\subsection{Capacitance sensing}

"Capacitance sensing" is a technique for measuring distances of nearby conductive objects by measuring the capacitance between the sensor and the object and uses a transmitter and a receiver electrode (Figure 1). When the transmitter is excited by a wave signal (of typically several hundred kilohertz), the receiver receives this wave. The magnitude of the receiving signal is proportional to the frequency and voltage of the transmitted signal, as well as to the capacitance between the two electrodes.

When a conductive object is close to both electrodes, it also capacitively couples to the electrode and strengthens the receiving wave signal amplitude. When a conductive and grounded object is close to both electrodes, it capacitively couples to the electrodes, drains the wave signal, and thus weakens the received signal amplitude. By measuring these effects, it is possible to detect the proximity of conductive objects.

The received signal often contains noises from nearby electric circuits and inverters of fluorescent lamps. To accurately measure signals from the transmitter electrode only, a technique called "lock-in amplifier" can be used. This technique uses an analogue switch as a phase-sensitive detector. A control signal is used to switch it on and off, to select signals that have the synchronized frequency and phase of the transmitted signal. Normally, a control signal needs to be created by phase-locking the incoming signal, but for capacitive sensing, the system can simply use a transmitted signal, because the transmitter and the receiver are both on the same circuit board.

This capacitive sensing technique is mainly used for proximity and position sensors [15]. In our work, capacitive sensing is used for measuring the arm shape by placing both the transmitter and the receiver electrodes on a wristband, and for measuring finger positions by attaching electrodes on the inside of clothes. 

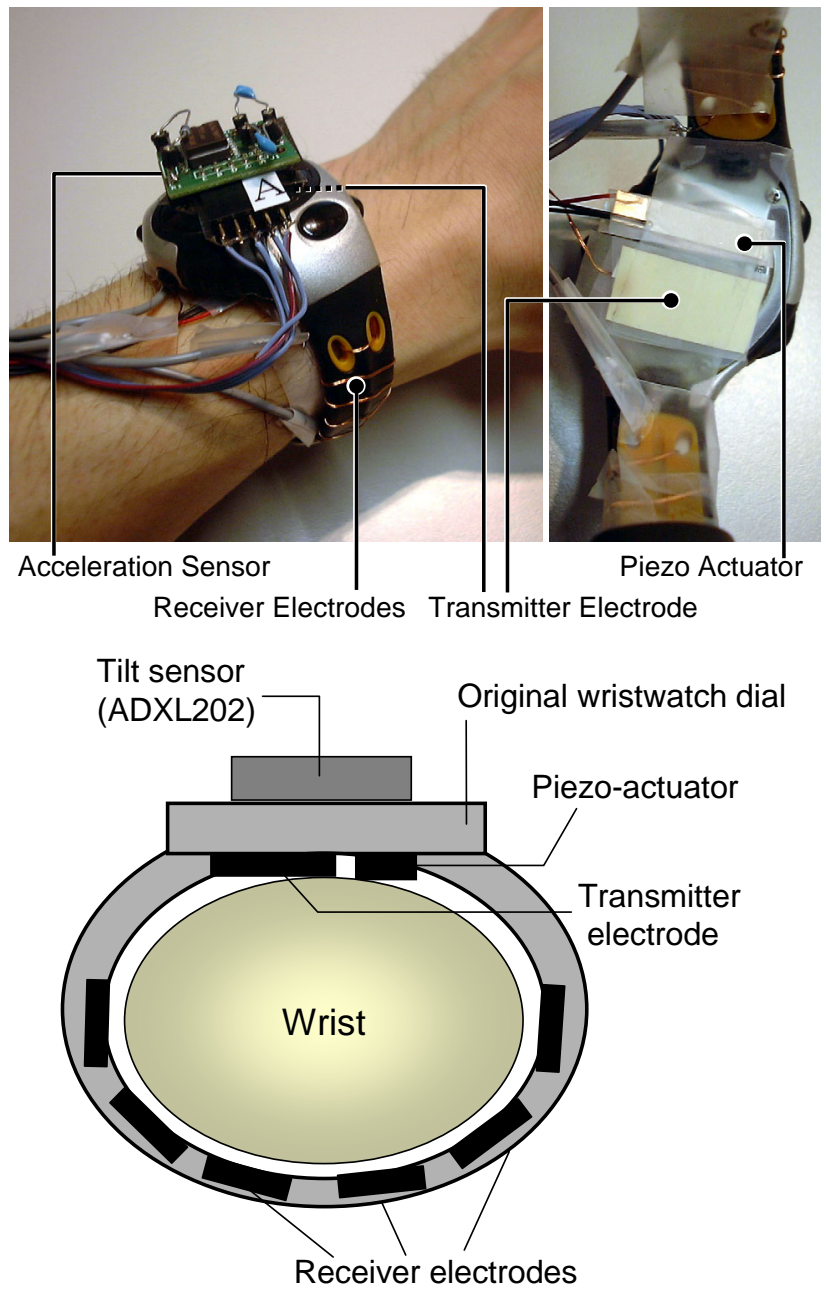

Figure 2: GestureWrist: Wristband-type input device.

\subsection{On-body networking}

Based also on capacitive sensing, a technique that transmits data through the human body has been proposed [14, 5]. Here, both a transmitter and a receiver are capacitively coupled to the human body. When a transmission signal is modulated by data (by using amplitude shift keying (ASK) or frequency shift keying (FSK)), this affects the modified signal that is received at the receiver side. Using this technology, wearable devices can communicate with each other [14], or they can automatically authenticate digital devices that are touched [5]. We also use this technique for distinguishing a wearer from other people while interacting with GesturePad.

\section{GestureWrist: A wristband-type input de- vice}

GestureWrist is a wristwatch-type input device that recognizes human hand gestures by capacitively measuring wrist-shape changes and also measuring forearm movements.
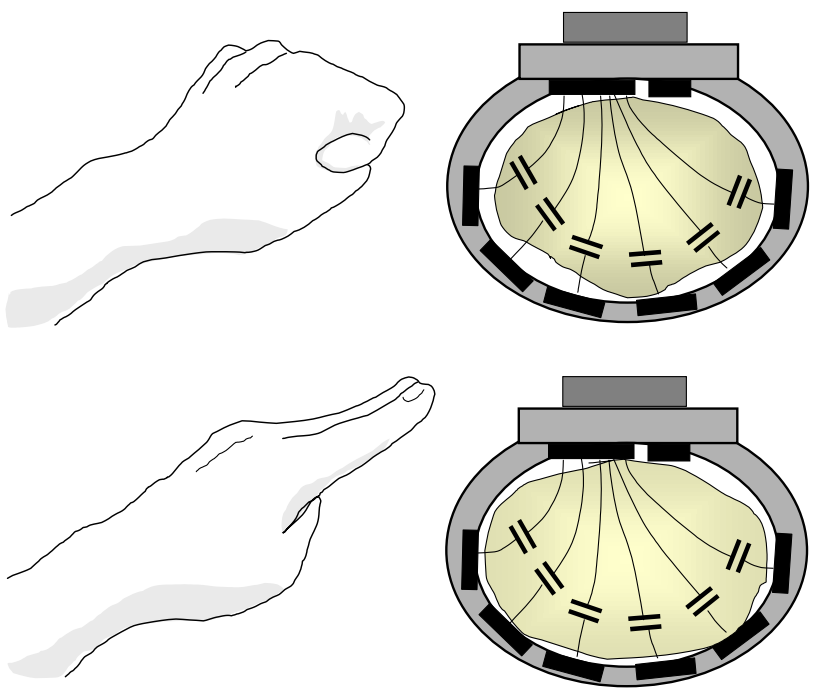

Figure 3: Sensing arm-shape change based on capacitive sensing.

Figure 2 shows the current GestureWrist prototype. This device consists of two input sensors (capacitance and acceleration sensors), and one tactile feedback actuator. The prototype is fabricated by attaching the sensors and actuators to a conventional wristwatch. We expect that embedding all the sensing elements within the wristwatch and the wristwatch band is technically possible, so a wearer can use this system in any social situation. Sensed information is processed at an external signal-processing board connected by a cable.

\subsection{Hand-gesture recognition}

GestureWrist recognizes hand gestures by measuring the changes of the arm shape on the inside of the wristband. To do this, a combination of transmitter and receiver electrodes are attached to the back of the watch dial and inside of the wristband. As described in the previous section, this combination acts as a capacitance sensor.

The principle of gesture sensing is shown in Figure 3. When a wearer opens and closes his or her hand, the crosssectional shape of the wrist changes accordingly; particularly, the left and right parts around the forearm sinew slightly bulge or cave in. A transmitter behind the wristband dial transmits a square wave signal (at approximately $160 \mathrm{KHz}$ ). This signal goes through the wrist, and is received by the receiver electrodes on the wristband. The amplitude of the receiving signal is determined by the capacitance between the transmitter electrode and the wrist, the resistance of the wrist, and the capacitance between the wrist and the receiver electrode. Since the first two values are mostly stable, the received signal strength is mainly determined by the last parameter (capacitance between the wrist and the receiver).

To calibrate the displacement of receiving electrodes, more than one electrode is installed on the wristband. The current prototype has three receivers. Each transmitterreceiver pair produces sensed values. The values conform 

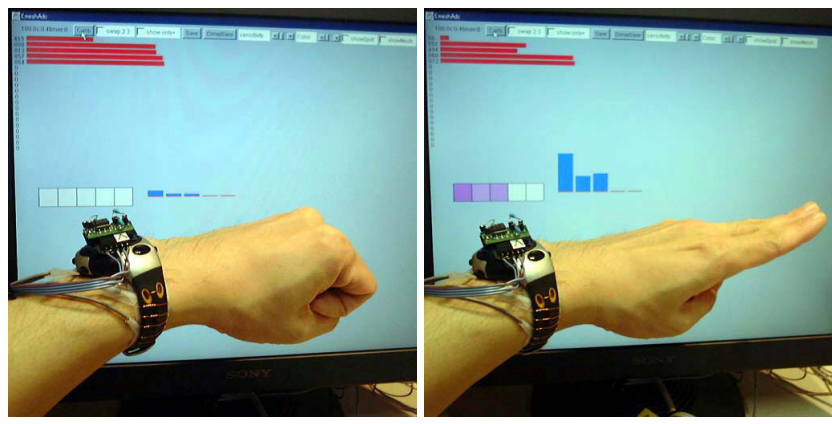

Figure 4: Relation between hand shape and obtained values.

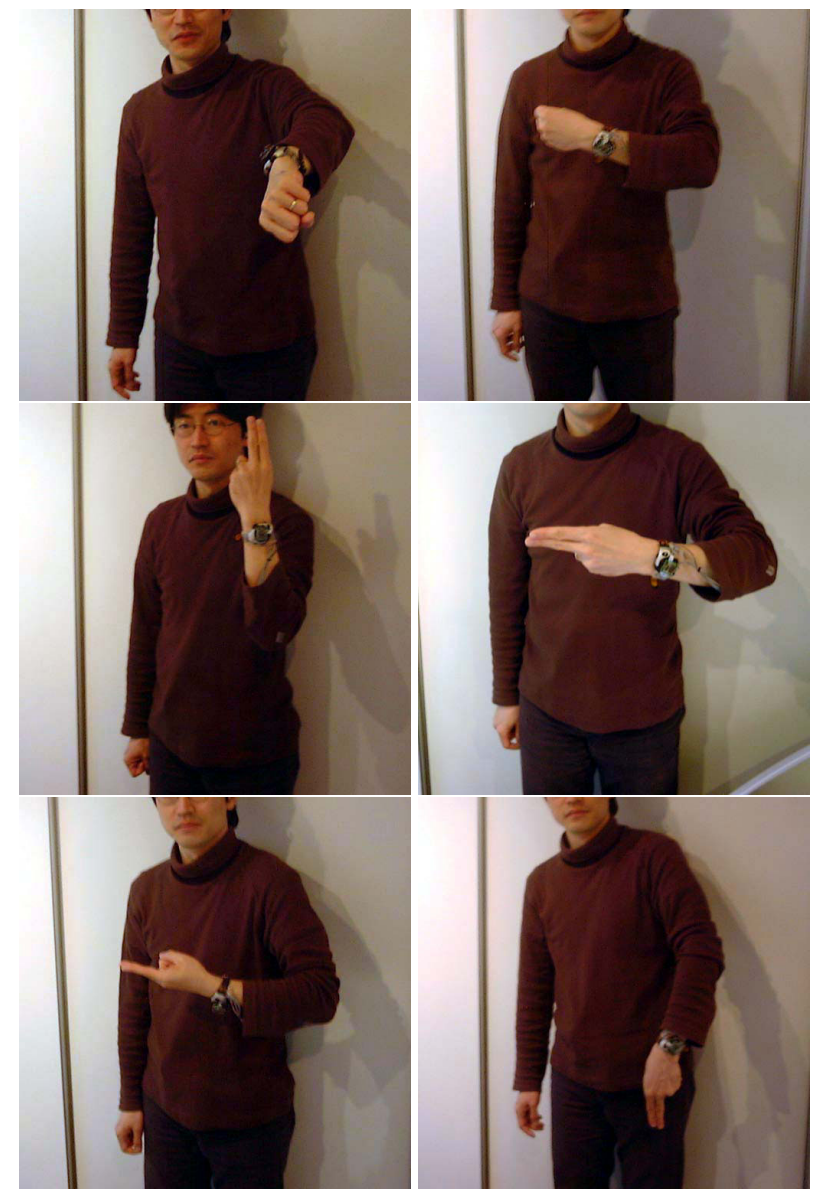

Figure 5: Example gesture commands

to a vector space (three dimensional, in this case), and a point in this space corresponds to a hand shape.

Figure 4 shows measured sensor values and their corresponding hand shapes. As shown here, the system can distinguish two hand shapes, grasping and pointing clearly.

\subsection{Forearm movement measurement}

In addition to the hand-shape measurement, an acceleration sensor (Analog Devices ADXL202) is mounted on the
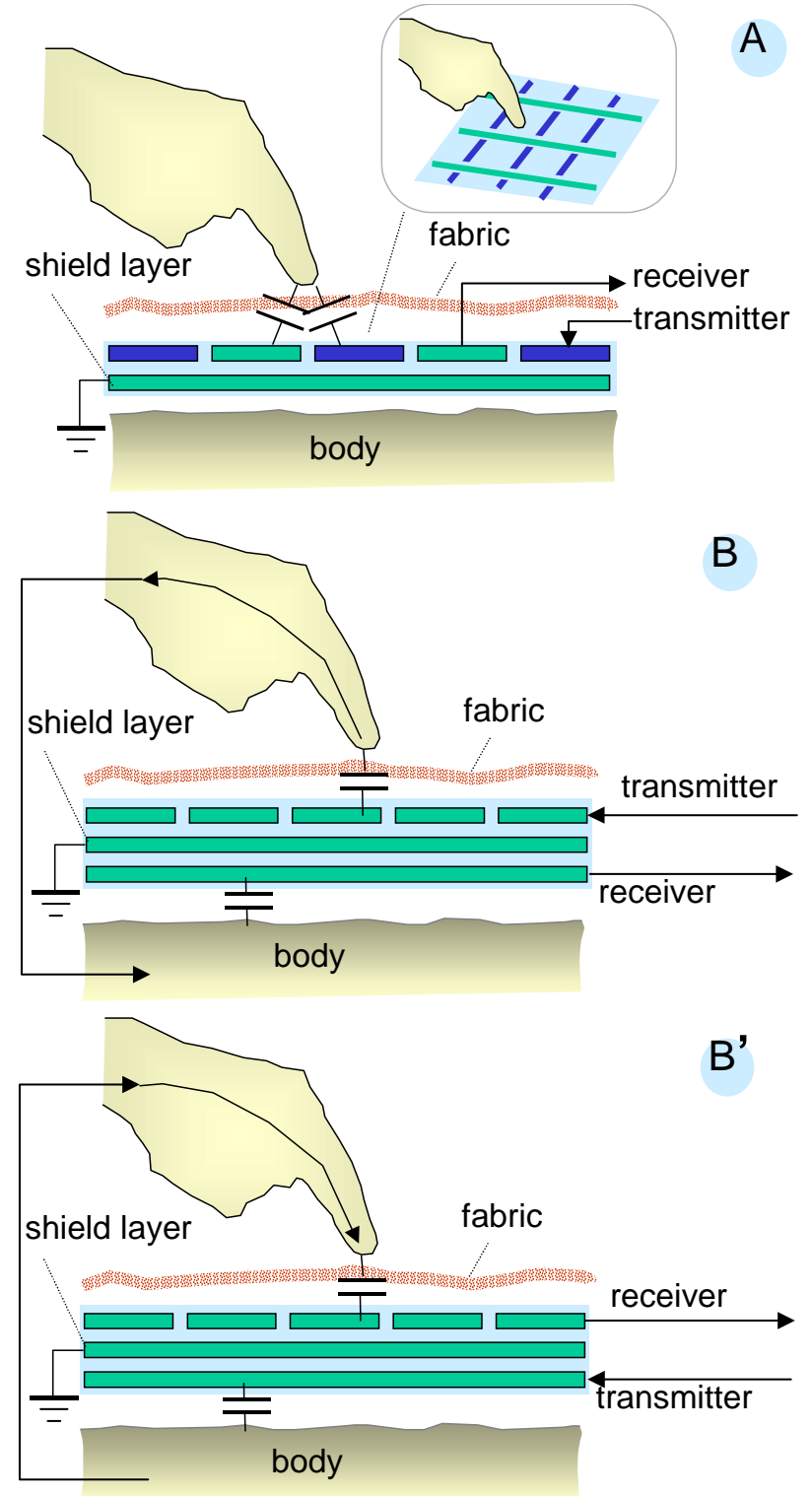

Figure 6: Sensor configurations for GesturePad

wristwatch dial. This sensor is a solid-state 2-axis sensor and measures the inclination of the forearm.

\subsection{Tactile feedback}

When a gesture is recognized, the GestureWrist gives feedback to the user by tactile sensation. On the inside of the wristwatch dial, a ceramic piezoelectric-actuator is attached to produce the feedback. We use $20-\mathrm{Hz}$ squarewave signals to excite this actuator.

\subsection{Combining two sensor inputs}

By combining these two inputs, we designed simple gesture commands. We selected two hand shapes (making a fist and pointing) and six different arm positions (palm 


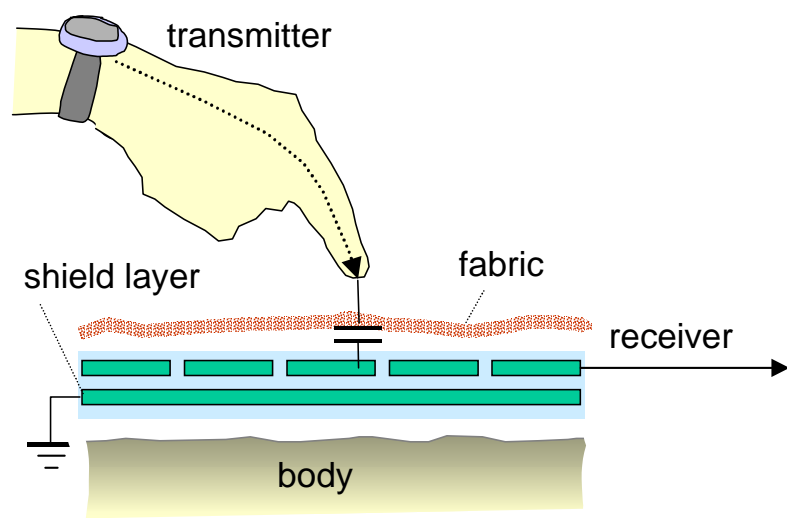

Figure 7: Variation of GesturePad Type-B which is used in combination with GestureWrist. This module receives a signal from the GestureWrist through the body.

up, palm right, palm left, palm down, forearm up, and forearm down). The hand shapes are used to separate gesture commands into segments, and two consecutive arm positions (e.g., palm left $\rightarrow$ palm down) make up one input command. Examples of gesture commands are shown in Figure 5.

Continuously adjust parameters is also possible by twisting the forearm. For example, a user can first decide which parameter to change, and control it by rotating his or her forearm.

Based on our experience, absolute values from capacitive sensors gradually change over a certain time period. This is mainly because the position of the wristband moves over time. On the other hand, the derivative of the capacitive values reflects the hand motion (e.g., from grasping to pointing) consistently. We are currently integrating this feature for to add stability and robustness to gesture recognition.

\section{GesturePad: A sensor module for interac- tive clothing}

Our next trial is to transform conventional clothes into interactive objects. Previous work on interactive clothes [7], have used metallic yarns woven into fabrics. This approach requires specially designed clothes, and is difficult to apply to clothes that already exist. We chose a "retrofit" approach that allows users to attach interactive modules to clothes easily. In addition, we particularly concentrated on making the attachment as unnoticeable as possible. We believe that clothes are a highly social media, and thus attaching obtrusive devices (such as [10]) is not an ideal solution.

The GesturePad, is a module that consists of a layer of sensors that can be attached to the inside of clothes. A wearer can control this module from the outside. As a result, a part of the clothes becomes interactive without changing its appearance.

\subsection{Sensor configurations}

Figure 6 shows three configurations of the GesturePad. All types can be attached to the clothes on the inside, and

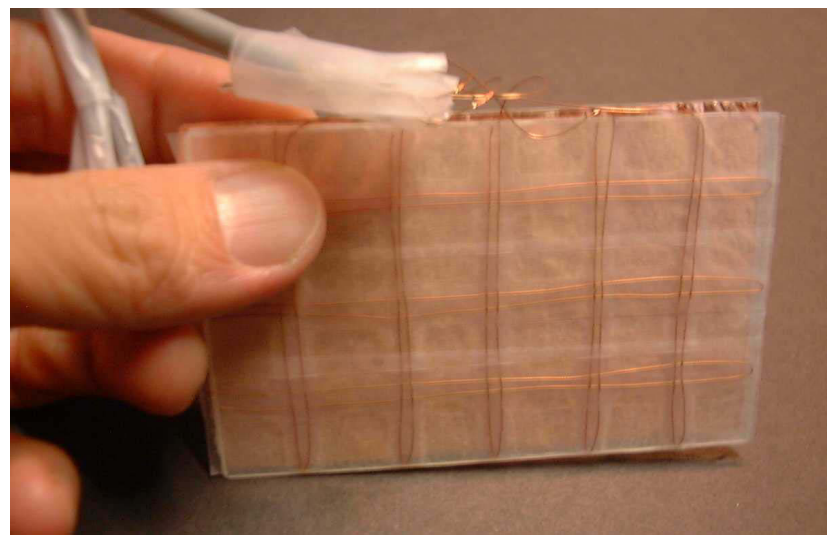

Figure 8: GesturePad prototype.

the wearer controls it from the outside.

Figure 6-(A), shows Type-A, which consists of an array of capacitive sensors (a combination of transmitters and receivers) and a shield layer attached to the behind. Each vertical grid line is a transmitter and each horizontal line a receiver electrode. The sensing of both the transmitter and the receiver is time-multiplexed, so the sensor can independently measure the capacitance value of each electrode crossing point.

When a user's finger is close enough to the sensor surface (typically within $1 \mathrm{~cm}$ ), the sensor grid recognizes the finger position. During this operation, the shield layer attached on the backside of the module blocks influence from the wearer's body. For example, when a module is placed on the inside of a lapel, a finger stroke gesture on the lapel becomes an input to the computer. This could enable controlling the volume of a worn MP3 player. Multiple sensor points on the module also enable multiple finger inputs. For example, a chording-keyboard type input would also be possible.

Figure 6-(B) and (B') show another sensor structure, Type-B (and B'), that consists of a transmitter and a receiver layer separated by a shield layer. In this configuration, a signal from the transmitter layer is capacitively coupled to a receiver layer through the user's body (i.e., on-body network). When the user's finger is within proximity of the GesturePad, a wave signal from the transmitter electrode is transmitted to the receiver one. This type could be put in a trouser pocket and operated from the outside of the pocket. One benefit of this configuration is that it can prevent other people from interacting with the sensor.

The Type-B(B') can also use an array of sensor electrodes so the user's finger motion is detected by comparing the received signal amplitudes. The difference between $B$ and $\mathrm{B}^{\prime}$ is the placement of transmitter and receiver electrodes. The Type-B places multiple transmitter electrodes on the front side and one receiver on the backside, while Type-B' uses multiple receiver electrodes on the front side. Since multiple transmitters can be easily implemented by time-multiplexing single transmitter, the needed hardware for Type-B is smaller than that of Type-B'.

Our current prototype for this Type-B integrates a trans- 

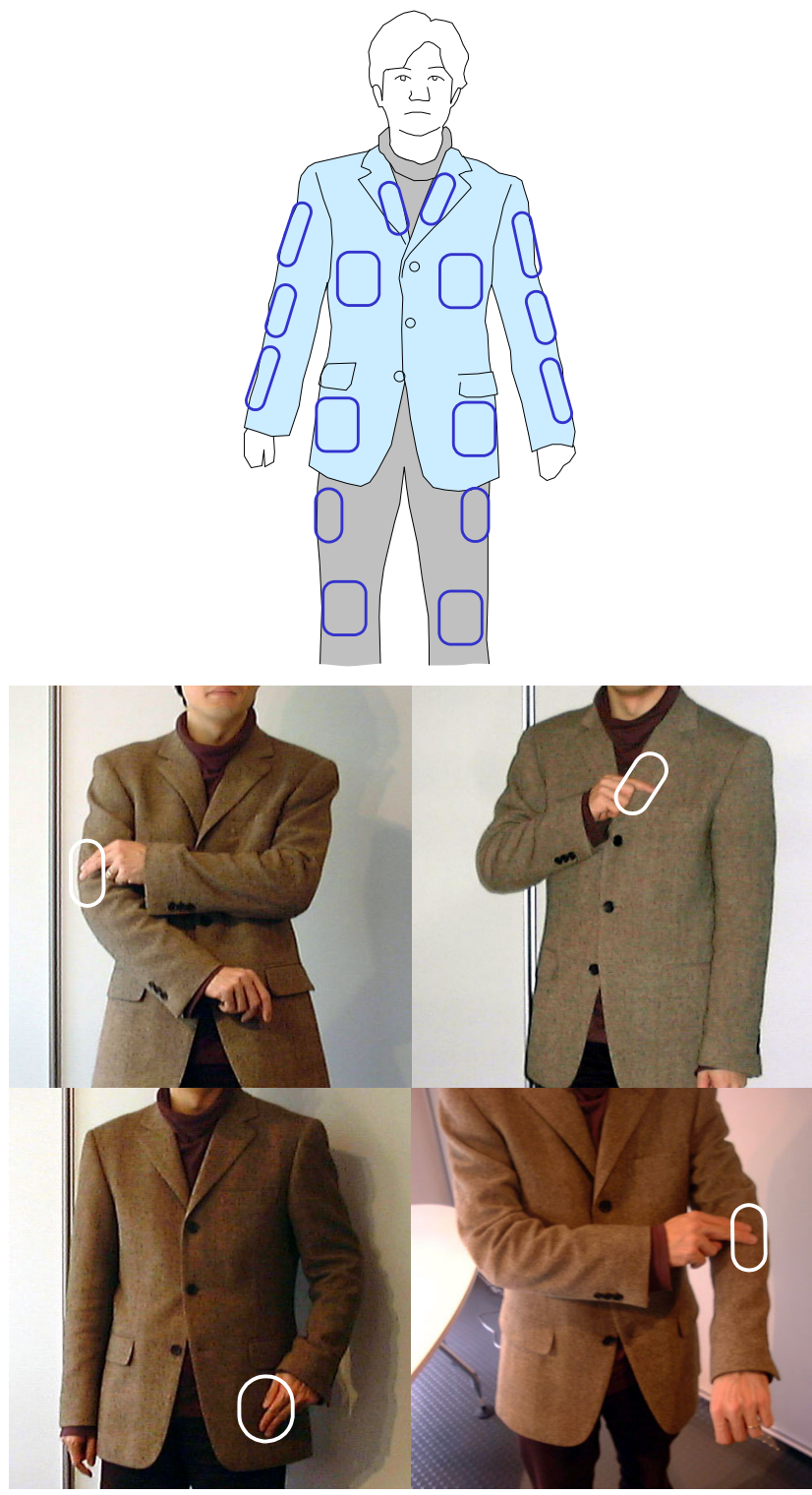

Figure 9: Possible input areas on interactive clothes.

mitter and a receiver into a single module, but separating these two is also possible. For example, the GestureWrist could work as a transmitter, and several receiver modules could be attached to various parts of the clothes (Figure 7).

\subsection{Interaction areas on clothing}

We have developed several variations of GesturePad and tried to attach them to different clothes parts. Figure 9 shows possible areas. Potentially, several parts could be assigned as input areas and used in combination. Supposedly one person using GesturePad is giving a presentation, he or she could select a command by touching a specific area of the jacket during the presentation (e.g., the left lapel for the "next slide" command, and the right lapel for "previous slide"). He or she may also control parameter values (e.g., selecting an appropriate position of a video) by sliding a finger up and down on the left sleeve. For the user's convenience, frequently used commands could be assigned to easily accessible areas, such as lapels or sleeves, while rarely used commands could be assigned to elbows or knees. These overall-body placements should also help users to remember commands. Combination of these actions would express more complicated commands, like the way baseball coach sends complicated block-signs to players by using a combination of body gestures.

Traditional input devices, such as mice, require changing the operation mode or selecting a menu item before actually performing an operation. This step is performed naturally when screen graphics are used interactively. However, when interacting with wearable computers, where the display capability might not be as good as that of a desktop computer, this step is troublesome. Assigning clothes areas and combining them (either sequentially or in parallel) would solve this problem.

\subsection{Clothes as communication surfaces}

Another feature of GesturePad is its ability to communicate with other digital devices. In this case, the same grid lines can be used as in the GesturePad sensor, in addition to near-field communication, but they are multiplexed by changing their frequencies $(160 \mathrm{KHz}$ for GesturePad, $38 \mathrm{KHz}$ for near-field communication).

A simple ID transmission device was developed to test this function. The device only consists of a microprocessor, which directly generates an ASK-modulated signal 2400 bps by using software. It feeds the signal to the attached electrode. When the module is in the detection area of the GesturePad, the GesturePad's receiver electrodes receive the transmitted signal. The received data is processed as an input to the wearable computer.

This capability can typically be applied to control data communication between digital devices nearby. For example, a user can perform a "pick-and-drop" operation [8], an interactive technique for moving digital data by physically picking it up and dropping it elsewhere. A user first "picks up" the data with a portable device and then "drops it" onto one sleeve. A corresponding data ID is transmitted from the device to the sleeve so the user virtually attaches the data to the clothing. Similarly, when a user first places a digital device, such as an MP3 player, to a sleeve, a wireless connection between the two is established and the sleeve acts as a controller for the MP3 player. This way the user might control the volume by sliding a finger up and down on the sleeve.

\section{Discussion and future research}

In this paper we introduced two input technologies for wearable computers called GestureWrist and GesturePad. Both devices are designed to be as unobtrusive as possible, so people can control wearable computers in any social situation. We expect that these technologies will not hinder performing other tasks because the devices do not change the appearance of conventional clothing or accessories. The technologies are also unobtrusive to their wearers, because they do not interfere with their daily activities. 
This work is still at an early stage, and we are considering developing several future applications. We plan to research the following areas in the immediate future.

\subsection{Combination with output devices}

In this paper we focus on input devices and do not clearly describe what kind of output devices could be used besides tactile feedback used by GestureWrist. The output devices should also be designed from the viewpoint of social acceptance and comfortability. An eyeglasses display with holographic optics [4] is one option, and we are also interested in combining an audio feedback function with GestureWrist and/or GesturePad. For example, if a GestureWrist / GesturePad user was also putting on a small earphone, the user could access audio information by interacting with GestureWrist / GesturePad. Combining audio information with wearable input devices should enable navigating freely through command hierarchies.

Another possibility is to use tactile feedback more aggressively. For example, if a GesturePad responds to a user with an attached actuator, it would work as a "virtual button" or "virtual slider". A user could feel this tactile feedback by "operating" both the fingers or the body; when a user slides the finger up or down on the sleeve, it feels as if an actual slider were mounted on the sleeve.

\subsection{Combination with on-body networks}

Currently, our prototype input devices communicate with the host computer through wires connections. We expect that these input devices can be combined with on-body networks, such as Personal Area Networks (PAN) [14], or the Touchnet [5], where the human body itself is used as a data communication medium.

We are currently particularly interested in applying an on-body network module called "wearable key", which is a wristwatch identification device [5].

\subsection{Sensing other parts of the body}

Similar to GestureWrist, measuring the shape of a part of the body as an input command should also be possible. For example, a sensor mounted on eyeglasses could measure eye motion without relying on traditional eyecameras. Simple eye-blink detection could be used for controlling wearable computers.

\section{ACKNOWLEDGEMENTS}

We thank the members of the Sony CSL Interaction Laboratory for helping us explore the ideas and technologies described in this paper. We remain indebted to Mario Tokoro and Toshi Doi for their continuing support of the research.

\section{References}

[1] Thomas Baudel and Michel Beaudouin-Lafon. Charade: remote control of objects using free-hand gestures. Communication of the ACM, 36(7):28-35, July 1993.

[2] HandeyKey Corporation. Twiddler home page. www.handykey.com.
[3] Masaaki Fukumoto and Yoshinobu Tonomura. Body coupled FingerRing: wireless wearable keyboard. In CHI'97 Proceedings, pages 147-154, 1997.

[4] Ichiro Kasai, Yasushi Tanijiri, Takeshi Endo, and Hiroak Ueda. A forgettable near eye display. In Proceedings of the Fourth International Symposium on Wearable Computers (ISWC'O0), pages 00-00, 2000.

[5] Nobuyuki Matsushita, Shigeru Tajima, Yuji Ayatsuka, and Jun Rekimoto. Wearable Key: Device for personalizing nearby environment. In Proceedings of the Fourth International Symposium on Wearable Computers (ISWC'00), pages 00-00, 2000.

[6] John Kangchunn Perng, Brian Fisher, Seth Hollar, and Kristofer S. J. Pister. Acceleration sensing glove (ASG). In The Third International Symposium on Wearable Computers (ISWC'99), pages 178-180, 1999.

[7] E. Rehmi Post and Margaret Orth. Smart Fabric, or "Wearable Clothing". In Proceedings of the First International Symposium on Wearable Computers (ISWC'97), pages 167-168, 1997.

[8] Jun Rekimoto. Pick-and-Drop: A Direct Manipulation Technique for Multiple Computer Environments. In Proceedings of UIST'97, pages 31-39, October 1997.

[9] T. Starner, J. Auxier, D. Ashbrook, and M. Gandy. The gesture pendant: A self-illuminating, wearable, infrared computer vision system for home automation control and medical monitoring. In Proceedings of the Fourth International Symposium on Wearable Computers (ISWC'00), pages 00-00, 2000.

[10] Bruce Thomas, Karen Grimmer, Dan Mackovec, Joanne Zucco, and Bernard Gunther. Determination of placement of a body-attached mouse as a pointing input device for wearable computers. In The Third International Symposium on Wearable Computers (ISWC'99), pages 193-194, 1999.

[11] Inc. Virtual Technologies. CyberGlove. www.virtex.com.

[12] John G. Webster, editor. Medical Instrumentation: Application and Design. John Wiley \& Sons, Inc., 1998.

[13] T.G. Zimmerman and J. Lanier. A hand gesture interface device. In Proceedings of CHI and Graphics Interface 1987, pages 189-192, 1987.

[14] Thomas Zimmerman. Personal area networks: Nearfield intrabody communication. IBM Systems Journal, 35(3-4):609-617, 1996.

[15] Thomas G. Zimmerman, Joshua R. Smith, Joseph A. Paradiso, David Allport, and Neil Gershenfeld. Applying electric field sensing to human-computer interfaces. In Proc. of CHI'95, pages 280-287, 1995. 